\title{
ON THE DIMENSIONLESSNESS OF INVARIANT SETS
}

\author{
L. OLSEN \\ Department of Mathematics, University of St. Andrews, St. Andrews, Fife KY16 9SS, Scotland \\ e-mail:lo@st-and.ac.uk
}

(Received 28 August, 2002; accepted 8 November, 2002)

\begin{abstract}
Let $M$ be a subset of $\mathbb{R}$ with the following two invariance properties: (1) $M+k \subseteq M$ for all integers $k$, and (2) there exists a positive integer $l \geq 2$ such that $\frac{1}{l} M \subseteq M$. (For example, the set of Liouville numbers and the Besicovitch-Eggleston set of non-normal numbers satisfy these conditions.) We prove that if $h$ is a dimension function that is strongly concave at 0 , then the $h$-dimensional Hausdorff measure $\mathcal{H}^{h}(M)$ of $M$ equals 0 or infinity.
\end{abstract}

2000 Mathematics Subject Classification. 28A80.

1. Introduction and statement of results. A dimension function $h$ is an increasing function $h:[0, \infty) \rightarrow[0, \infty)$ with $h(0)=0$. If $h$ is a dimension function, we shall denote the $h$-dimensional Hausdorff measure of a subset $E$ of $\mathbb{R}$ by $\mathcal{H}^{h}(E)$; the reader is referred to [5] for the definition of $\mathcal{H}^{h}(E)$. If $t>0$ and $h$ equals the power function $h(r)=r^{t}$, then we shall write $\mathcal{H}^{h}(E)=\mathcal{H}^{t}(E)$. Recall that a dimension function $h$ is called an exact dimension function of a subset $E$ of $\mathbb{R}$ if $\mathcal{H}^{h}(E)$ is positive and finite. Finally, a subset $E$ of $\mathbb{R}$ is called dimensionless if it does not have an exact dimension function, i.e. if $\mathcal{H}^{h}(E)$ equals 0 or infinity for all dimension functions $h$.

In this paper we show that if a subset $M$ of the real line satisfies two rather weak invariance conditions shared by many naturally occurring sets (for example, the set of Liouville numbers and the Besicovitch-Eggleston set of non-normal numbers satisfy these invariance conditions), then the $h$-dimensional Hausdorff measure of $M$ equals 0 or infinity for a large class of dimension functions $h$.

Observe that if a dimension function $h$ is concave in a neighbourhood of 0 , then

$$
\liminf _{r \searrow 0} \frac{h(\lambda r)}{\lambda h(r)} \geq 1
$$

for all $\lambda \in(0,1)$. In this paper we consider dimension functions which satisfy a slightly stronger condition. We shall say that a dimension function $h$ is strongly concave at 0 if

$$
\liminf _{r \searrow 0} \frac{h(\lambda r)}{\lambda h(r)}>1
$$

for all $\lambda \in(0,1)$. We shall now give some examples of dimension functions that are strongly concave at 0 .

(1) Power functions $h(r)=r^{t}$ with $t \in(0,1)$ are strongly concave at 0 .

(2) Recall that a continuous function $L:[0, \infty) \rightarrow[0, \infty)$ with $L(r)>0$ for all $r>0$ is called slowly varying if $\lim _{r \searrow 0} \frac{L(\lambda r)}{L(r)}=1$ for all $\lambda>0$. Functions of the form 
$h(r)=r^{t} L(r)$, where $t \in(0,1)$ and $L:[0, \infty) \rightarrow[0, \infty)$ is a slowly varying function, are strongly concave at 0 .

(3) The dimension function $h$ defined by $h(r)=\frac{1}{\log \frac{1}{r}}$ for $r \in(0,1)$ and $h(0)=0$ is strongly concave at 0 .

We can now state the main result of this paper.

THEOREM 1. Let $M$ be a subset of $\mathbb{R}$ satisfying the following two invariance conditions:

(1) $M+k \subseteq M$, for all integers $k$;

(2) there exists a positive integer $l \geq 2$ such that $\frac{1}{l} M \subseteq M$.

Then $\mathcal{H}^{h}(M)=0$ or $\mathcal{H}^{h}(M)=\infty$, for all dimension functions $h$ that are strongly concave at 0 .

The proof of Theorem 1 is given in Section 2.

REMARK 1. If a subset $M$ of $\mathbb{R}$ satisfies condition (1) in Theorem 1; i.e. if $M+k \subseteq$ $M$ for all integers $k$, then in fact $M+k=M$, for all integers $k$. Indeed, for all integers $k$ we have $M=(M-k)+k \subseteq M+k$ since $M-k \subseteq M$.

REMARK 2. If a non-empty subset $M$ of $\mathbb{R}$ satisfies conditions (1) and (2) in Theorem 1, i.e. if $M+k \subseteq M$ for all integers $k$ and there exists a positive integer $l \geq 2$ such that $\frac{1}{l} M \subseteq M$, then $M$ is dense in $\mathbb{R}$. Indeed, let $x \in \mathbb{R}$ and $r>0$. Since $M$ is non-empty there exists $t \in M$. Next, choose integers $p$ and $q$ with $q \geq 1$ such that $\left|x-\frac{p}{l^{q}}\right| \leq \frac{r}{2}$ and $\frac{|t|}{l^{q}} \leq \frac{r}{2}$. Then clearly $\frac{p+t}{l^{q}} \in \frac{1}{l^{q}}(p+M) \subseteq \frac{1}{l^{q}} M \subseteq \frac{1}{l^{q-1}} M \subseteq \ldots \subseteq$ $M$ and $\left|x-\frac{p+t}{l^{q}}\right| \leq\left|x-\frac{p}{l^{q}}\right|+\frac{|t|}{l^{q}} \leq \frac{r}{2}+\frac{r}{2}=r$. This shows that $M$ is dense in $\mathbb{R}$.

Many naturally occurring sets of numbers satisfy the conditions in Theorem 1 . We shall now consider two examples.

EXAMPLE. The Liouville numbers. Let $\mathbb{\mathbb { }}$ denote the set of Liouville numbers, i.e.

$$
\begin{gathered}
\mathbb{L}=\{x \in \mathbb{R} \backslash \mathbb{Q} \mid \text { for all } n \in \mathbb{N} \text { there exist integers } p \text { and } q \\
\text { with } \left.q>1 \text { such that }\left|x-\frac{p}{q}\right|<\frac{1}{q^{n}}\right\} .
\end{gathered}
$$

It is well known that the Hausdorff dimension of $\mathbb{L}$ is 0 , cf. for example Oxtoby's book [6, Theorem 2.4] for a simple direct proof or [1, p. 69] for a proof based on Jarník's theorem. In particular, this implies that the $t$-dimensional Hausdorff measure $\mathcal{H}^{t}(\mathbb{L})$ of $\mathbb{L}$ equals 0 , for all $t>0$. It is therefore natural to ask whether or not $\mathbb{L}$ is dimensionless. It follows easily from the definition of the Liouville numbers that $\mathbb{L}+k \subseteq \mathbb{L}$ and $\frac{1}{k} \mathbb{L} \subseteq \mathbb{L}$, for all non-zero integers $k$, and, by applying Theorem 1 to $\mathbb{L}$, we obtain the following result.

THEOREM 2. Let $h$ be a dimension function that is strongly concave at 0 . Then $\mathcal{H}^{h}(\mathbb{L})=0$ or $\mathcal{H}^{h}(\mathbb{L})=\infty$.

EXAMPLE. The Besicovitch-Eggleston set of non-normal numbers. Let $N \geq 2$ be a fixed positive integer, and for $x \in \mathbb{R}$ let $x=[x]+\sum_{n=1}^{\infty} \frac{\varepsilon_{n}(x)}{N^{n}}$, where $[x] \in \mathbb{Z}$ and 
$\varepsilon_{n}(x) \in\{0,1, \ldots, N-1\}$, denote the unique non-terminating $N$-adic expansion of $x$. For each digit $i \in\{0,1, \ldots, N-1\}$, we write

$$
\Pi_{i}(x ; n)=\frac{\left|\left\{1 \leq k \leq n \mid \varepsilon_{k}(x)=i\right\}\right|}{n} ;
$$

so $\Pi_{i}(x ; n)$ denotes the frequency of the digit $i$ among the first $n$ digits in the $N$-adic expansion of $x$. For a given probability vector $\mathbf{p}=\left(p_{0}, p_{1}, \ldots, p_{N-1}\right)$, the BesicovitchEggleston set $B(\mathbf{p})$ is defined by

$$
B(\mathbf{p})=\left\{x \in \mathbb{R} \mid \Pi_{i}(x ; n) \rightarrow p_{i} \text { as } n \rightarrow \infty \text { for all } i\right\} .
$$

Besicovitch [2] and Eggleston [4] computed the Hausdorff dimension, $\operatorname{dim} B(\mathbf{p})$, of $B(\mathbf{p})$. In fact, they proved that $\operatorname{dim} B(\mathbf{p})=-\frac{\sum_{i} p_{i} \log p_{i}}{\log N}$; the reader is referred to the textbook [3, p. 142] for a contemporary proof of this result based on the ergodic theorem. It is natural to ask whether or not the Besicovitch-Eggleston set $B(\mathbf{p})$ is dimensionless. Since clearly $B(\mathbf{p})+k \subseteq B(\mathbf{p})$ for all integers $k$ and $\frac{1}{N} B(\mathbf{p}) \subseteq B(\mathbf{p})$, an application of Theorem 1 gives the following result.

THEOREM 3. Let $h$ be a dimension function that is strongly concave at 0 . Then $\mathcal{H}^{h}(B(\mathbf{p}))=0$ or $\mathcal{H}^{h}(B(\mathbf{p}))=\infty$.

In fact, using the law of the iterated logarithm (rather than relying on the invariance properties of the set $B(\mathbf{p}))$, Smorodinsky [7] proved the following stronger version of Theorem 3: $\mathcal{H}^{h}(B(\mathbf{p}))=0$ or $\mathcal{H}^{h}(B(\mathbf{p}))=\infty$ for all concave dimension functions.

2. Proof of Theorem 1. We shall now prove Theorem 1 . We first state and prove an auxiliary result. For a dimension function $h$ and a positive real number $s$ write

$$
\underline{d}_{h}(s)=\liminf _{r \searrow 0} \frac{h(s r)}{h(r)}
$$

and

$$
\bar{d}_{h}(s)=\limsup _{r \searrow 0} \frac{h(s r)}{h(r)} .
$$

Proposition 4. Let $h$ be a dimension function and let $f: \mathbb{R}^{n} \rightarrow \mathbb{R}^{n}$ be a similarity with ratio equal to $s>0$; i.e. $|f(x)-f(y)|=s|x-y|$, for all $x, y \in \mathbb{R}^{n}$. Then

$$
\underline{d}_{h}(s) \mathcal{H}^{h}(E) \leq \mathcal{H}^{h}(f(E)) \leq \bar{d}_{h}(s) \mathcal{H}^{h}(E),
$$

for all $E \subseteq \mathbb{R}^{n}$

Proof. We write $|B|$ for the diameter of a subset $B$ of $\mathbb{R}^{n}$. For a positive real number $\delta$, we denote the $\delta$ approximative $h$-dimensional Hausdorff measure by $\mathcal{H}_{\delta}^{h}$; the reader is referred to [5] for the definition of $\mathcal{H}_{\delta}^{h}$.

Part 1. We first prove that $\mathcal{H}^{h}(f(E)) \geq \underline{d}_{h}(s) \mathcal{H}^{h}(E)$. Let $\varepsilon>0$, and choose $r_{\varepsilon}>0$ such that $\frac{h(s r)}{h(r)} \geq \underline{d}_{h}(s)-\varepsilon$ for all $0<r<r_{\varepsilon}$. Next, fix $0<\delta<r_{\varepsilon}$, and let $\left(B_{i}\right)_{i}$ be an $s \delta$-cover of $f(E)$. Since $\left|f^{-1}\left(B_{i}\right)\right|=\frac{1}{s}\left|B_{i}\right|$, we conclude that $\left(f^{-1}\left(B_{i}\right)\right)_{i}$ is a $\delta$-cover of $E$. 
Hence

$$
\begin{aligned}
\sum_{i} h\left(\left|B_{i}\right|\right) & =\sum_{i} \frac{h\left(\left|B_{i}\right|\right)}{h\left(\frac{1}{s}\left|B_{i}\right|\right)} h\left(\left|f^{-1}\left(B_{i}\right)\right|\right) \geq \sum_{i}\left(\underline{d}_{h}(s)-\varepsilon\right) h\left(\left|f^{-1}\left(B_{i}\right)\right|\right) \\
& \geq\left(\underline{d}_{h}(s)-\varepsilon\right) \mathcal{H}_{\delta}^{h}(E) .
\end{aligned}
$$

This implies that $\mathcal{H}_{s \delta}^{h}(f(E)) \geq\left(\underline{d}_{h}(s)-\varepsilon\right) \mathcal{H}_{\delta}^{h}(E)$ for all $0<\delta<r_{\varepsilon}$. Letting first $\delta \searrow 0$ and then letting $\varepsilon \searrow 0$ gives $\mathcal{H}^{h}(f(E)) \geq \underline{d}_{h}(s) \mathcal{H}^{h}(E)$.

Part 2. Next we prove that $\mathcal{H}^{h}(f(E)) \leq \bar{d}_{h}(s) \mathcal{H}^{h}(E)$. Let $\varepsilon>0$, and choose $r_{\varepsilon}>0$ such that $\frac{h(s r)}{h(r)} \leq \bar{d}_{h}(s)+\varepsilon$ for all $0<r<r_{\varepsilon}$. Next, fix $0<\delta<r_{\varepsilon}$, and let $\left(B_{i}\right)_{i}$ be an $\delta$-cover of $E$. Since $\left|f\left(B_{i}\right)\right|=s\left|B_{i}\right|$, we conclude that $\left(f\left(B_{i}\right)\right)_{i}$ is an $s \delta$-cover of $f(E)$. Hence

$$
\begin{aligned}
\sum_{i} h\left(\left|B_{i}\right|\right) & =\sum_{i} \frac{h\left(\left|B_{i}\right|\right)}{h\left(s\left|B_{i}\right|\right)} h\left(\left|f\left(B_{i}\right)\right|\right) \geq \sum_{i} \frac{1}{\bar{d}_{h}(s)+\varepsilon} h\left(\left|f\left(B_{i}\right)\right|\right) \\
& \geq \frac{1}{\bar{d}_{h}(s)+\varepsilon} \mathcal{H}_{s \delta}^{h}(f(E)) .
\end{aligned}
$$

This implies that $\left(\bar{d}_{h}(s)+\varepsilon\right) \mathcal{H}_{\delta}^{h}(E) \geq \mathcal{H}_{s \delta}^{h}(f(E))$ for all $0<\delta<r_{\varepsilon}$. Letting first $\delta \searrow 0$ and then letting $\varepsilon \searrow 0$ gives $\mathcal{H}^{h}(f(E)) \leq \bar{d}_{h}(s) \mathcal{H}^{h}(E)$.

We can now prove Theorem 1 .

Proof of Theorem 1. It follows from the assumptions on $M$ that $M \cap[0,1)=$ $(M \cap[k, k+1))-k$, for all integers $k$, and Proposition 4 therefore implies that

$$
\mathcal{H}^{h}(M \cap[0,1))=\mathcal{H}^{h}(M \cap[k, k+1)) .
$$

Hence, it suffices to prove that $\mathcal{H}^{h}(M \cap[0,1))$ equals 0 or infinity. Write $I=[0,1)$, and for $k=0,1, \ldots, l-1$ put $I_{k}=\left[\frac{k}{l}, \frac{k+1}{l}\right)$. Also define maps $f_{k}: I \rightarrow I_{k}$ by $f_{k}(x)=\frac{x+k}{l}$. Since $M+k \subseteq M$, for all integers $k$, and $\frac{1}{l} M \subseteq M$, we conclude that $f_{k}(M \cap I) \subseteq$ $M \cap I_{k}$, whence $\cup_{k=0}^{l-1} f_{k}(M \cap I) \subseteq M \cap I$. This implies that

$$
\begin{aligned}
\mathcal{H}^{h}(M \cap I) & \geq \mathcal{H}^{h}\left(\bigcup_{k=0}^{l-1} f_{k}(M \cap I)\right) \\
& =\sum_{k=0}^{l-1} \mathcal{H}^{h}\left(f_{k}(M \cap I)\right) \\
& \geq \sum_{k=0}^{l-1} \underline{d}_{h}\left(\frac{1}{l}\right) \mathcal{H}^{h}(M \cap I) \\
& =\underline{l}_{h}\left(\frac{1}{l}\right) \mathcal{H}^{h}(M \cap I) .
\end{aligned}
$$


However, since $h$ is strongly concave at 0 , we have

$$
l \underline{d}_{h}\left(\frac{1}{l}\right)=\liminf \operatorname{in}_{r \searrow 0} \frac{h\left(\frac{1}{l} r\right)}{\frac{1}{l} h(r)}>1 .
$$

It now follows from (1) and (2) that $\mathcal{H}^{h}(M \cap I)$ equals 0 or infinity.

\section{REFERENCES}

1. V. Bernik and M. Dodson, Metric Diophantine approximation on manifolds, Cambridge Tracts in Mathematics No. 137 (Cambridge University Press, 1999).

2. A. Besicovitch, On the sum of digits of real numbers represented in the dyadic system, Math. Ann. 110 (1934), 321-330.

3. P. Billingsley, Ergodic theory and information (John Wiley and Sons, 1965).

4. H. G. Eggleston, The fractional dimension of a set defined by decimal properties, Quart. J. Math., Oxford Ser. 20 (1949), 31-36.

5. K. J. Falconer, Fractal geometry-Mathematical foundations and applications (John Wiley and Sons, 1990).

6. J. Oxtoby, Measure and category (Springer Verlag, 1980).

7. M. Smorodinsky, Singular measures and Hausdorff measures, Israel J. Math. 7 (1969), 203-206. 